\title{
Omega-3 Fatty Acids Reduce Inflammation in Rat Apical Periodontitis
}

\author{
Mariane Maffei Azuma, DDS, MSc, PhD, *f João Eduardo Gomes-Filbo, DDS, MSc, PhD, * \\ Edilson Ervolino, DDS, MSc, PbD, ${ }^{\dagger}$ Carolina de Barros Moraes Cardoso, DDS, * \\ Camila Barbosa Pipa, DDS, * Toshibisa Kawai, DDS, PhD, ${ }^{\neq}$Leticia Citelli Conti, DDS, * \\ and Luciano Tavares Angelo Cintra, DDS, MSc, PbD*
}

\section{Ahstract}

Introduction: The effects of omega-3 polyunsaturated fatty acids ( $\omega-3$ PUFAs) on pro- and anti-inflammatory mediators were evaluated in a rat model of pulp exposure-induced apical periodontitis (AP). Methods: Twenty-eight male Wistar rats were divided into 4 groups: control, untreated rats (group $\mathrm{C}$ ); control rats treated with $\omega-3$ PUFAs (group C-0); rats with pulp exposure-induced AP (group AP); and rats with pulp exposure-induced AP treated with $\omega-3$ PUFAs (group AP-0). Omega-3 PUFAs were administered orally once a day for 15 days before pulp exposure; this treatment was continued for 30 days after pulp exposure. The rats were sacrificed 30 days after pulp exposure, and their dissected jaws were subjected to immunohistochemical analysis to detect immunoreactivity for tumor necrosis factor alpha (TNF- $\alpha$ ), interleukin (IL)-6, IL-1 $\beta$, IL-17, and IL-10 on the periapical bone surface. The results were statistically evaluated using analysis of variance and the Tukey post-test. The significance level was set at 5\%. Results: Immunoreactivity for the proinflammatory cytokines TNF- $\alpha$, IL-6, IL-1 $\beta$, and IL-17 was higher in the AP group than in the AP-O, C, and $\mathrm{C}-\mathrm{O}$ groups $(P<.05)$. Immunoreactivity for the antiinflammatory cytokine IL-10 was lower in the AP group than in the AP-O group $(P<.05)$. Conclusions: Supplementation with $\omega$-3 PUFAs can modulate the inflammatory response in rat AP, decreasing levels of TNF- $\alpha$, IL-6, IL-1 $\beta$, and IL-17 but increasing levels of IL-10. (J Endod 2018;44:604-608)

\section{Key Words}

Apical periodontitis, cytokine, endodontic infection, omega-3 fatty acid
$A_{\mathrm{sis}}^{\mathrm{p}}$ pical periodontitis (AP) is characterized by the presence of fibrous and granulated tissue as well as by infiltrates of various inflammatory cells ( $\mathrm{T}$ lymphocytes, B lymphocytes,

Significance
Our findings indicate that systemic oral administra-
tion with $\omega-3$ PUFAs can modulate the inflamma-
tion in rat apical periodontitis, decreasing TNF- $\alpha$,
IL-6, IL- $1 \beta$, and IL-17 and increasing IL-10 expres-
sion.
and macrophages) (1)

This periapical inflammation is an immune response against bacterial infection in necrotic pulp. When host defense mechanisms cannot eradicate the infection over time, chronic AP ensues, leading to destruction of the bone structures in the periapical area (2).

In AP, several proinflammatory cytokines are produced locally to mediate the immune response, and these promote bone resorption $(3,4)$. In particular, proinflammatory cytokines such as tumor necrosis factor alpha (TNF- $\alpha$ ), interleukin (IL) -6, IL-1 $\beta$, and IL-17 may stimulate osteoclastogenesis and bone resorption (5). Such "cytokine storms" are related to the development of AP $(4,5)$.

Conversely, some studies have suggested that IL-10, which can suppress the production of proinflammatory cytokines such as IL-1, IL-6, and TNF- $\alpha(6,7)$, slows AP development (5). This suggests that, to reduce bone loss during AP, researchers must develop therapeutic regimens that can down-regulate the proinflammatory response and increase the anti-inflammatory response.

Omega-3 polyunsaturated fatty acids ( $\omega-3$ PUFAs), such as eicosapentaenoic acid (EPA) and docosahexaenoic acid (DHA), have been studied as an adjuvant therapy to treat oral diseases, including gingivitis (8), periodontitis (9-11), AP (12), and recurrent aphthous stomatitis (13). Related findings have indicated that diets supplemented with $\omega-3$ PUFAs inhibit the production of arachidonic acid metabolites via the cyclooxygenase 2 and lipoxygenase pathways, thus reducing the proinflammatory response (14) and decreasing inflammatory symptoms (13). Another study involving pulp exposure-induced AP in rats showed that $\omega-3$ PUFAs reduce bone resorption and promote bone generation, leading to a decrease in tartrate-resistant acid phosphatase-positive cells and an increase in osteocalcin-positive cells (12). However, to our knowledge, no study has ever investigated the effects of $\omega$-3 PUFAs on cytokine expression in either human AP patients or animal models of endodontic infection.

Therefore, the present study aimed to evaluate the effects of an $\omega$-3 PUFA-supplemented diet on pathogenic bone resorption in a rat model of pulp exposure-induced

From the Departments of *Endodontics, and ${ }^{\dagger}$ Basic Sciences, Araçatuba Dental School, Universidade Estadual Paulista (UNESP), Araçatuba, São Paulo, Brazil; ${ }^{\ddagger}$ Department of Periodontology, NOVA Southeastern University College of Dental Medicine, Fort Lauderdale, Florida; ${ }^{\S}$ Department of Cariology, Restorative Sciences and Endodontics, University of Michigan, Ann Arbor, Michigan.

Address requests for reprints to Dr Luciano Tavares Angelo Cintra, Department of Endodontics, Araçatuba School of Dentistry, UNESP-Universidade Estadual Paulista, Araçatuba, São Paulo, Brazil. E-mail address: lucianocintra@foa.unesp.br 0099-2399/\$ - see front matter

Copyright (C) 2017 American Association of Endodontists. https://doi.org/10.1016/j.joen.2017.12.008 


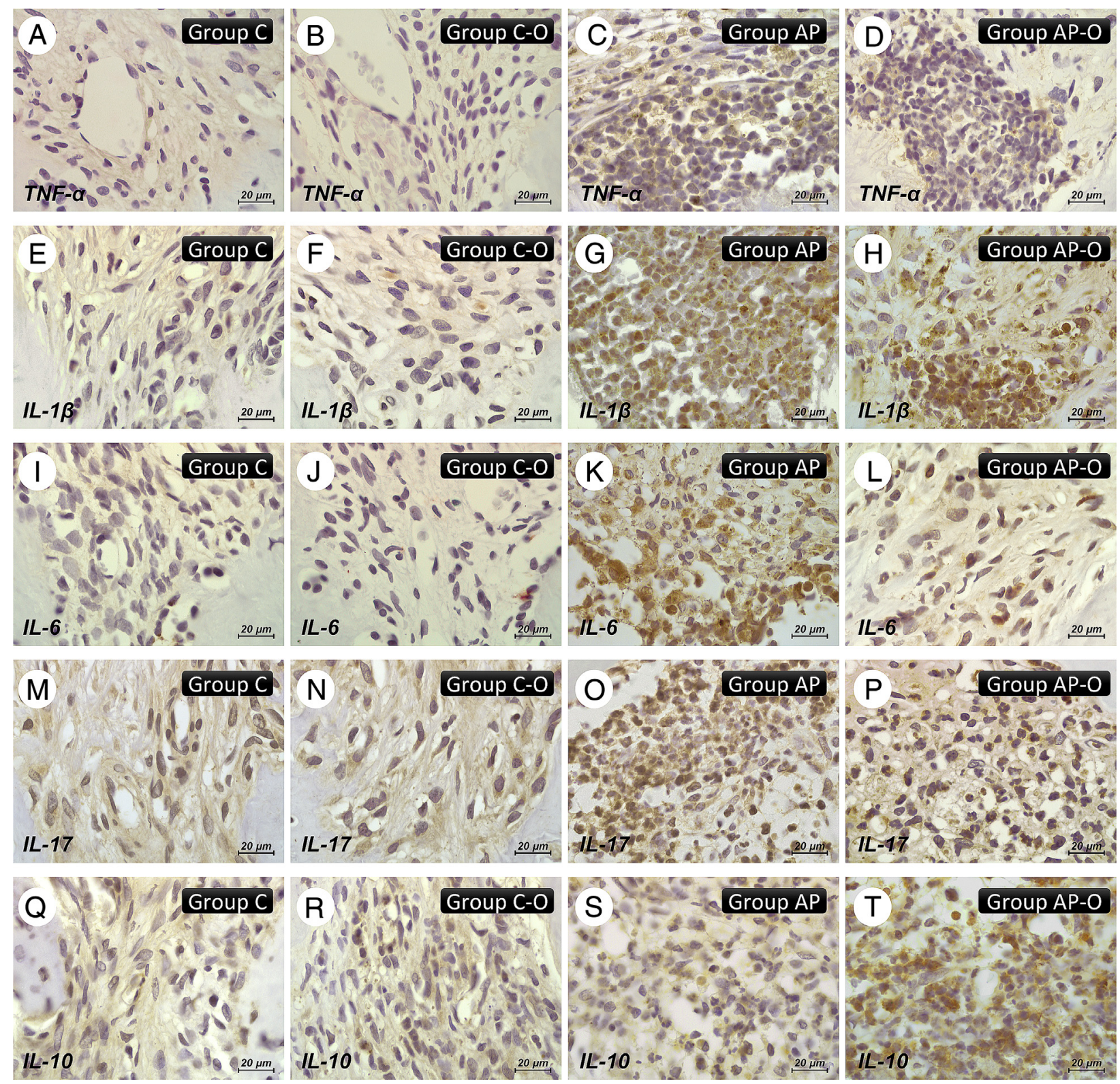

Figure 1. Immunoreactivity of TNF- $\alpha$, IL-1 $\beta$, IL-6, IL-17, and IL-10 in the periapical bone 30 days after pulp exposure. Representative images of the TNF- $\alpha$ immunoreaction among the groups: $(A)$ group $\mathrm{C},(B)$ group $\mathrm{C}-0,(C)$ group AP, and $(D)$ group AP-0. Representative images of the IL-1 $\beta$ immunoreaction among the groups: $(E)$ group C, $(F)$ group C-0, $(G)$ group AP, and $(H)$ group AP-0. Representative images of the IL-6 immunoreaction among the groups: $(I)$ group C, $(J)$ group C-0, $(K)$ group AP, and $(L)$ group AP-0. Representative images of the IL-17 immunoreaction among the groups: $(M)$ group C, $(N)$ group C-0, $(O)$ group AP, and $(P)$ group AP-0. Representative images of IL-10 immunoreaction among the groups: $(Q)$ group C, $(R)$ group C-O, $(S)$ group AP, and $(T)$ group AP-O. Original magnification: $400 \times$

AP. To accomplish this, we used immunohistochemical analysis to monitor the effects of $\omega$-3 PUFAs on the production of the proinflammatory cytokines TNF- $\alpha$, IL-1 $\beta$, IL- 6 , and IL-17 and the antiinflammatory cytokine IL-10 in rat periapical tissues.

\section{Experimental Animals}

\section{Materials and Methods}

The experimental protocol was approved by the Institutional Ethics Committee (CEUA 2014-00550) of the Universidade Estadual Paulista, São Paulo, Brazil, and it was conducted in accordance with relevant guidelines. Six-week-old male Wistar rats (Rattus norvegicus albinus) ( $n=28$ ), weighing 200-250 g each, were used in this study. The rats were housed in mini-isolators equipped with the Ventilife sys- tem (Alesco, São Paulo, SP, Brazil), which injects air at a continuous, low-speed flow. In this way, the system maintains ideal air exchange and internal pressure, isolating the animals from external influences. The rats were maintained in temperature-controlled rooms and given ad libitum access to water and food.

The rats were randomly assigned to 4 groups of 7 animals each: control, untreated rats (group C); control rats treated with $\omega$-3 PUFAs (group C-0); rats with pulp exposure-induced AP (group AP); and rats with pulp exposure-induced AP treated with $\omega-3$ PUFAs (group AP-0).

\section{Supplementation with $\omega-3$ PUFAs}

For 15 days before AP induction (prophylactic administration) and 30 days afterward (therapeutic administration), rats in the C-O 

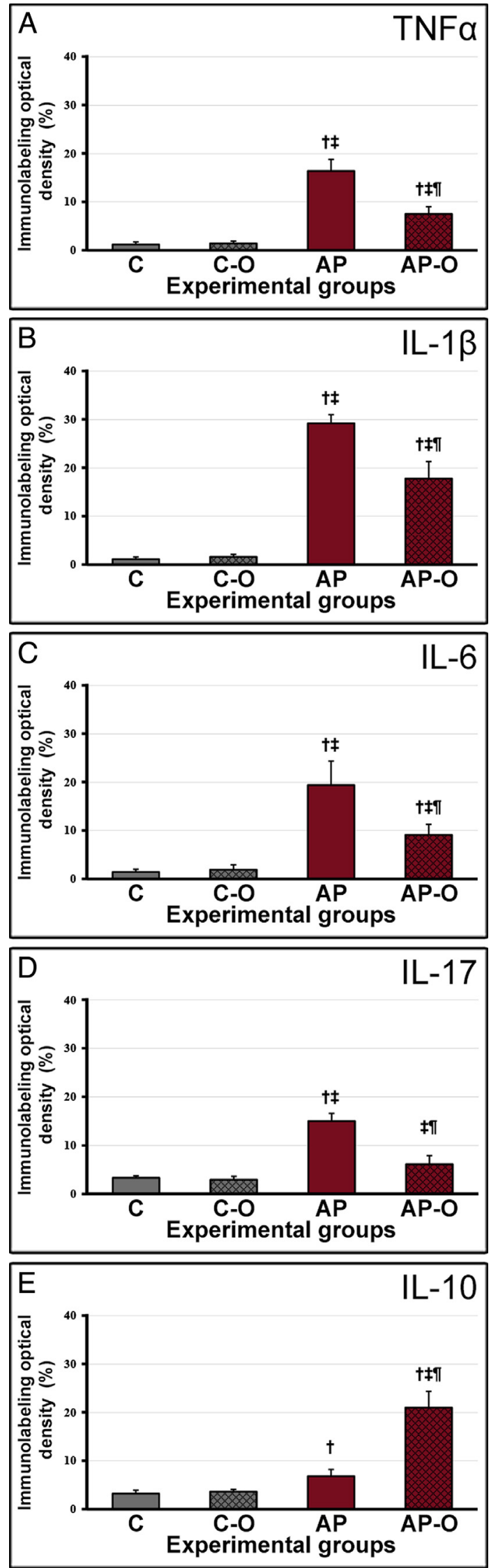

Figure 2. Immunolabeling optical density for $(A)$ TNF- $\alpha,(B)$ IL-1 $\beta,(C)$ IL-6, $(D)$ IL-17, and $(E)$ IL-10 in the periapical tissue in different and AP-0 groups were administered $\omega$-3 PUFAs (Omega-3; Catarinense Pharma-Laboratório Catarinense SA, Joinville, SC, Brazil) once daily via oral gavage (solution in distilled water, $40 \mathrm{mg} / \mathrm{kg}$; $60 \% \mathrm{EPA}$ and $40 \%$ $\mathrm{DHA})$. Those in the $\mathrm{C}$ and $\mathrm{AP}$ groups received distilled water over the same period. Thus, the rats were treated with either $\omega$-3 PUFAs or control water for a total of 45 days $(9,12,15)$.

\section{Induction of AP}

Rats were anesthetized with ketamine $(87 \mathrm{mg} / \mathrm{kg}$ Francotar; Virbac do Brazil Industria e Comercio Ltda, São Paulo, SP, Brazil) and xylazine (13 mg/kg Rompun; Bayer SA, São Paulo, SP, Brazil). In the AP and AP-0 groups, AP was induced by exposing the pulp of the right mandibular first molars using surgical round burs (Broca LN Long Neck; Dentsply Industria e Comercia Ltda, Petrópolis, SP, Brazil). The coronal pulp tissue was exposed to the oral cavity in this way for 30 days $(12,16,17)$.

\section{Immunohistologic Analyses}

The mandibles of the sacrificed rats were decalcified in 10\% EDTA for 30 days and subjected to conventional histologic processing to produce paraffin-embedded tissue sections. Semiserial sections $(4 \mu \mathrm{m})$ were performed in a laterolateral direction, which ensured that the mandibular first molar was sectioned in its longitudinal axis. Immunohistochemical processing followed the protocol described by Garcia et al (18).

The histologic slides were divided into 5 batches, each of which contained samples from all groups and was incubated with 1 of the following primary antibodies: goat anti-TNF- $\alpha$ (1:100 SC 1348; Santa Cruz Biotechnology, Santa Cruz, CA), rabbit anti-IL-1 $\beta$ (1:100 SC 7884, Santa Cruz Biotechnology), rabbit anti-IL-6 (1:200 SC 1265, Santa Cruz Biotechnology), goat anti-IL-10 (1:100 SC 1783, Santa Cruz Biotechnology), and rabbit anti-IL-17 (1:200 SC 7927, Santa Cruz Biotechnology). To visualize the immunolabeling, biotinylated secondary antibodies were applied for 2 hours followed by a streptavidin-horseradish peroxidase conjugate for 1 hour (Universal Dako-Labeled Streptavidin-Biotin Kit; Dako Laboratories, Carpinteria, CA). Chromogen 3,3'-diaminobenzidine tetrahydrochloride (DAB Chromogen Kit, Dako Laboratories) was used to detect immunolabeling. As a negative control, the specimens were submitted to the same procedures but without the primary antibody.

Immunohistochemical analyses were performed by a certified histologist (E.E.) who was blinded to the experimental groups. One histologic section from each animal was used with each antibody. Brownish coloring in the cytoplasm and extracellular matrix denoted immunolabeling. The region of interest was a $1200 \times 800-\mu \mathrm{m}$ area located within the periapical tissues of the mandibular first molar's mesial root. Images from this region were obtained at $400 \times$ magnification under guidance from image analysis software (Axiovision 4.8.2; Carl Zeiss, Gottingen, Germany). To determine the immunolabeling optical density, the AP area was delineated using the brownish-color threshold tool (19). This value was expressed as a random unit percentage of the optical density (mean \pm standard deviation).

\section{Statistical Analysis}

Statistical analysis and data tabulation were performed using SigmaPlot software (Systat Software Inc, San Jose, CA). The immunolabeling optical density data were submitted to the Shapiro-Wilk test,

experimental groups. $\uparrow$ Statistically significant difference in relation to group C. $\ddagger$ Statistically significant difference in relation to group C-0. IStatistically significant difference in relation to group AP. 
analysis of variance, and the Tukey post-test. The significance level was set at $5 \%$.

\section{Results}

To evaluate the effects of $\omega$-3 PUFAs on pro- and anti-inflammatory cytokine expression in AP, cytokine immunoreactivity was measured. Figure 1 shows photomicrographs of the TNF- $\alpha$, IL-6, IL-1 $\beta$, IL-17, and IL-10 immunoreactivity patterns, and Figure 2 shows the immunolabeling optical density for these cytokines.

Tooth sections stained for TNF- $\alpha$ lacked immunoreactive cells completely in the $\mathrm{C}$ (Fig. $1 A$ ) and C-O (Fig. 1B) groups (Fig. 2A), and the AP group (Fig. $1 C$ ) showed higher immunoreactivity than the AP-0 group (Fig. $1 D, P<.05)$ (Fig. $2 A$ ).

In the case of IL-1 $\beta$, the $\mathrm{C}$ (Fig. $1 E$ ) and $\mathrm{C}-0$ (Fig. $1 F$ ) groups lacked immunoreactivity completely (Fig. $2 B$ ), and the AP group (Fig. $1 G$ ) showed higher immunoreactivity than the AP-O group (Fig. $1 H, P<.05$ ) (Fig. $2 B$ ).

In the case of IL-6, the $\mathrm{C}$ (Fig. 1I) and C-O (Fig. 1J) groups lacked immunoreactivity completely (Fig. $2 C$ ), and the AP group (Fig. $1 K$ ) showed higher immunoreactivity than the AP-0 group (Fig. $1 L, P<.05$ ) (Fig. $2 C$ ).

In the case of IL-17, the $\mathrm{C}$ (Fig. $1 M$ ) and $\mathrm{C}-0$ (Fig. $1 N$ ) groups lacked immunoreactivity completely (Fig. 2D), and the AP group (Fig. 1O) showed higher immunoreactivity than the AP-O group (Fig. $1 P$, $P<.05$ ) (Fig. $2 D$ ). Conversely, IL-10 was not detected in the $\mathrm{C}$ (Fig. 1Q) and C-O (Fig. 1R) groups, and the AP group (Fig. 1S) showed lower immunoreactivity than the AP-0 group (Fig. 1T, $P<.05$ ) (Fig. $2 E$ ).

\section{Discussion}

In the present study, systemic oral administration of $\omega-3$ PUFAs decreased the expression of the proinflammatory cytokines TNF- $\alpha$, IL-1 $\beta$, IL- 6 , and IL-17 but increased that of the anti-inflammatory cytokine IL-10 in the context of AP. This study was conducted over a 30-day period, which is long enough to observe the development of chronic AP and complete pulpal necrosis $(12,17)$. We chose the chronic AP model to ensure there were no systemic signs of acute infection, such as abscesses, which can influence the expression of proinflammatory mediators in periapical tissues. Thus, we could evaluate the role of $\omega-3$ PUFAs in rats without significant variation in their AP. The influence of $\omega$-3 PUFAs on the acute phases of AP should still be investigated in the future.

This study corroborated a previous investigation showing that systemic oral administration of $\omega$-3 PUFAs suppresses bone resorption and promotes bone generation, thus decreasing osteoclastogenesis, increasing osteoblastogenesis, and promoting normal bone remodeling homeostasis (12). The present data also indicated that pro- and antiinflammatory cytokines are associated with osteoclastogenesis and osteoblastogenesis, respectively, in APs.

During AP formation and development, inflammatory cells (T cells, B cells, and macrophages) are recruited to the periapical tissues to eliminate infection. This results in a classic "cytokine storm" $(1,4)$ in which TNF- $\alpha$ promotes the sensitivity of mature osteoclasts to the receptor activator of nuclear factor kappa-B ligand (20). Together with its receptor, the receptor activator of nuclear factor kappa-B upregulates osteoclast formation, activation, and survival in a variety of pathologic bone resorption conditions, including AP (4).

In addition, TNF- $\alpha$, IL- 6 , and IL- $1 \beta$ act as proinflammatory mediators during bone resorption in AP (21), and IL-1 $\beta$, IL-6, and cyclooxygenase 2 are regulated by nuclear factor-kappa beta activation (22). Importantly, EPA and DHA can inhibit TNF- $\alpha$ production by preventing nuclear factor-kappa beta activation $(23,24)$. Therefore, in the present study, the expression of TNF- $\alpha$, IL- $1 \beta$, and IL- 6 may have been reduced by $\omega-3$ PUFAs via NF- $\kappa$ B modulation.
Another study using Porphyromonas gingivalis-induced experimental periodontal disease in rats showed that $\omega-3$ PUFA supplementation decreased the expression of TNF- $\alpha$, IL- $1 \beta$, and IL- 6 in periodontal tissues (25). The present report corroborated these results. In addition, although several studies have reported on IL-17 expression in AP $(26,27)$, none have confirmed its role in the development of disease. IL-17 appears to exacerbate chronic inflammation, and it plays a role in cyst formation (26). In the present study, we found that supplementation with $\omega$-3 PUFAs decreased IL-17 expression in AP. Therefore, we hypothesize that DHA suppresses the activity of IL-17-producing CD4+ T cells, as reported previously (28). Nonetheless, using rats supplemented with $\omega$-3 PUFAs, more studies should be performed to elucidate the role of IL-17 in AP.

IL-10 is an anti-inflammatory cytokine that can regulate B-cell proliferation and differentiation (29). Moreover, as a suppressor of bone loss in periodontal disease (30) and AP (31), it directly affects osteoclastogenesis. In the present study, we found that IL-10 can suppress bone loss in rat AP and that IL-10 expression was similar among the $\mathrm{C}, \mathrm{C}-\mathrm{O}$, and AP groups. This finding corroborates previous studies that found no differences in IL-10 expression among wild-type mice with AP (30). However, we found higher IL-10 expression in the AP0 group than in the AP group $(P<.05)$. This result contradicts previous studies that found no differences in IL-10 expression among individuals with periodontal disease regardless of $\omega$-3 PUFA supplementation (25). This discrepancy could be attributed to differences in dietary composition or experimental time. Studies have further demonstrated that anti-inflammatory effects associated with $\omega-3$ PUFAs are dose and time dependent (32).

The findings presented here indicate that systemic oral administration of $\omega$-3 PUFAs can modulate the inflammation in rat AP, decreasing TNF- $\alpha$, IL-6, IL-1 $\beta$, and IL-17 and increasing IL-10 expression. Future studies should explore the capacity of $\omega-3$ PUFAs to generate endogenous metabolites that promote inflammation resolution, such as resolvins, docosatrienes, and neuroprotectins. Previous studies have shown this to occur in other inflammatory diseases $(14,33)$, and the present study supports further investigation into $\omega$-3 PUFAs as a treatment for AP.

\section{Acknowledgments}

Supported by FAPESP (grant no. 2013/26390-0).

The authors declare no conflicts of interest related to this study.

\section{References}

1. Liapatas S, Nakou M, Rontogianni D. Inflammatory infiltrate of chronic periradicular lesions: an immunohistochemical study. Int Endod J 2003;36:464-71.

2. Gazivoda D, Dzopalic T, Bozic B, et al. Production of proinflammatory and immunoregulatory cytokines by inflammatory cells from periapicallesions in culture. J Oral Pathol Med 2009;38:605-11.

3. Siqueira JF Jr, Rocas IN. Bacterial pathogenesis and mediators in apical periodontitis. Braz Dent J 2007;18:267-80.

4. Silva MJ, Kajiva M, AlShwaimi E, et al. Bacteria-reactive immune response may induce RANKL-expressing $\mathrm{T}$ cells in the mouse periapical bone loss lesion. J Endod 2012:38:346-50.

5. Araujo-Pires AC, Francisconi CF, Biguetti CC, et al. Simultaneous analysis of T helper subsets (Th1, Th2, Th9, Th17, Th22, Tfh, Tr1 and Tregs) markers expression inperiapical lesions reveals multiple cytokine clusters accountable for lesions activity and inactivity status. J Appl Oral Sci 2014;22:336-46.

6. Houri-Haddad Y, Soskolne WA, Halabi A, et al. IL-10 gene transfer attenuates P. gingivalis-induced inflammation. J Dent Res 2007;86:560-4.

7. Mosser DM, Zhang X. Interleukin-10: new perspectives on an old cytokine. Immunol Rev 2008;226:205-18. 
8. Araghizadeh N, Paknejad M, Alaeddini M, et al. The efficacy and prophylactic characteristics of omega-3 fatty acids in experimental gingivitis in rats. Iran J Basic Med Sci 2014;17:87-92.

9. Kesavalu L, Vasudevan B, Raghu B, et al. Omega-3 fatty acid effect on alveolar bone loss in rats. J Dent Res 2006;85:648-52.

10. Vardar-Sengul S, Buduneli E, Turkoglu 0, et al. The effects of selective COX-2 inhibitor/celecoxib and omega-3 fatty acid on matrix metalloproteinases, TIMP-1, and laminin-5gamma2-chain immunolocalization in experimental periodontitis. J Periodontol 2008;79:1934-41.

11. Deore GD, Gurav AN, Patil R, et al. Omega 3 fatty acids as a host modulator in chronic periodontitis patients: a randomised, double-blind, placebo-controlled, clinical trial. J Periodontal Implant Sci 2014;44:25-32.

12. Azuma MM, Gomes-Filho JE, Ervolino E, et al. Omega 3 fatty acids reduce bone resorption while promoting bone generation in rat apical periodontitis. J Endod 2017;43:970-6.

13. El Khouli AM, El-Gendy EA. Efficacy of omega-3 in treatment of recurrent aphthous stomatitis and improvement of quality of life: arandomized, double-blind, placebocontrolled study. Oral Surg Oral Med Oral Pathol Oral Radiol 2014;117:191-6.

14. Calder PC. The relationship between the fatty acid composition of immune cells and their function. Prostaglandins Leukot Essent Fatty Acids 2008;79:101-8.

15. Vardar-Sengül S, Buduneli N, Buduneli E, et al. Dietary supplementation of omega-3 fatty acid and circulating levels of interleukin-1beta, osteocalcin, and C-reactive protein in rats. J Periodontol 2006;77:814-20.

16. Cintra LT, Samuel RO, Azuma MM, et al. Multiple apical periodontitis influences serum levels of cytokines and nitric oxide. J Endod 2016;42:747-51.

17. Cintra LT, da Silva Facundo AC, Prieto AK, et al. Blood profile and histology in oral infections associated with diabetes. J Endod 2014;40:1139-44.

18. Garcia VG, Knoll LR, Longo M, et al. Effect of the probiotic Saccharomyces cerevisiae on ligature-induced periodontitis in rats. J Periodontal Res 2016;51:26-37.

19. King TW, Brey EM, Youssef AA, et al. Quantification of vascular density using a semiautomated technique for immunostained specimens. Anal Quant Cytol Histol 2002;24:39-48.

20. Weitzmann MN, Pacifici R. The role of T lymphocytes in bone metabolism. Immunol Rev 2005;208:154-68.

21. Martinho FC, Chiesa WM, Leite FR, et al. Correlation between clinical/radiographic features and inflammatory cytokine networks produced bymacrophages stimulated with endodontic content. J Endod 2012;38:740-5.
22. Mullen A, Loscher CE, Roche HM. Anti-inflammatory effects of EPA and DHA are dependent upon time and dose-response elements associated with LPS stimulation in THP-1-derived macrophages. J Nutr Biochem 2010;21: 444-50.

23. Novak TE, Babcock TA, Jho DH, et al. NF- $\kappa$ B inhibition by $\omega-3$ fatty acids modulates LPS-stimulated macrophage TNF- $\alpha$-transcription. Am J Physiol Lung Cell Mol Physiol 2003;284:L84-9.

24. Vedin I, Cederholm T, Freund Levi Y, et al. Effects of docosahexaenoic acid-rich $\mathrm{n}-3$ fatty acid supplementation on cytokine release from blood mononuclear leukocytes: the OmegAD study. Am J Clin Nutr 2008;87:1616-22.

25. Kesavalu L, Bakthavatchalu V, Rahman MM, et al. Omega-3 fatty acid regulates inflammatory cytokine/mediator messenger RNA expression in Porphyromonas gingivalis-induced experimental periodontal disease. Oral Microbiol Immunol 2007;22:232-9.

26. Ajuz NC, Antunes H, Mendonça TA, et al. Immunoexpression of interleukin 17 in apical periodontitis lesions. J Endod 2014;40:1400-3.

27. Yang S, Zhu L, Xiao L, et al. Imbalance of interleukin-17+ T-cell and Foxp3+ regulatory T-cell dynamics in rat periapical lesions. J Endod 2014;40:56-62.

28. Kong W, Yen JH, Ganea D. Docosahexaenoic acid prevents dendritic cell maturation, inhibits antigen-specific Th1/Th17 differentiation and suppresses experimental autoimmune encephalomyelitis. Brain Behav Immun 2011;25:872-82.

29. Volk H, Asadullah K, Gallagher G, et al. IL-10 and its homologs: important immune mediators and emerging immunotherapeutic targets. Trends Immunol 2001;22: 414-7.

30. Sasaki H, Okamatsu Y, Kawai T, et al. The interleukin-10 knockout mouse is highly susceptible to Porphyromonas gingivalis-induced alveolar bone loss. J Periodontal Res 2004;39:432-41.

31. De Rossi A, Rocha LB, Rossi MA. Interferon-gamma, interleukin-10, Intercellular adhesion molecule-1, and chemokine receptor 5, but not interleukin-4, attenuate the development of periapical lesions. J Endod 2008;34:31-8.

32. Kremer JM, Lawrence DA, Jubiz W, et al. Dietary fish oil and olive oil supplementation in patients with rheumatoid arthritis. Clinical and immunologic effects. Arthritis Rheum 1990;33:810-20.

33. Chapkin RS, Kim W, Lupton JR, et al. Dietary docosahexaenoic and eicosapentaenoic acid: emerging mediators of inflammation. Prostaglandins Leukot Essent Fatty Acids 2009;81:187-91. 